\title{
DNA methylation: Basic biology and application to traumatic brain injury
}

\author{
Mr. Bilal A. Mateen ${ }^{1^{*}}$ \\ BSc \\ bilalmateen@hotmail.co.uk \\ Dr. Ciaran S. Hill ${ }^{*}$ \\ MBBS, MRCS, MRCP \\ ciaranhill@gmail.com \\ Dr. Simon C. Biddie ${ }^{3}$ \\ MBChB, PhD \\ simon.c.biddie@gmail.com \\ Prof. David K. Menon'2,3,4,\# \\ MD, PhD, F.R.C.A., F.R.C.P., F.Med.Sci. \\ dkm13@cam.ac.uk
}

1 - Division of Medicine, University College London, London, WC1E 6BT, UK

Tel: +44 (0)20 7679 2000. Fax: +44 (0)20 76792000

2 - John van Geest Centre for Brain Repair, School of Clinical Medicine, Addenbrookes Hospital, Cambridge CB2 0SP, UK

Tel: +44 1223 331160. Fax: +44 1223331174

3 - Division of Anaesthesia, Department of Medicine, University of Cambridge, Cambridge, CB2 0SP, UK

Tel: +44 (0)1223 217889. Fax: +44 (0)1223 217887

4 - Wolfson Brain Imaging Centre, Department of Clinical Neurosciences, University of Cambridge, Cambridge, CB2 0QQ, UK

Tel: +44 (0)1223 331823. Fax: +44 (0)1223 331826

\# - Corresponding Author:

Prof. David K Menon

University Division of Anaesthesia

Addenbrooke's Hospital and University of Cambridge

Cambridge CB2 2QQ, UK

Email: dkm13@cam.ac.uk

Telephone: +44 (0)1223 217889

Fax: +44 (0)1223 217887

Key Words: Epigenetics, Traumatic Brain Injuries, DNA Methylation

Running Title: TBI and DNA Methylation

Table of Contents Title: DNA methylation: Basic biology and application to traumatic brain injury 


\section{$\underline{\text { Abstract }}$}

This article reviews the literature pertinent to epigenetic changes, and in particular DNA methylation following Traumatic Brain Injury (TBI). TBI is a heterogeneous disease that is a major cause of death and long-term disability. The links between TBI and epigenetics, the process by which environmental factors alter gene expression without changing the underlying DNA sequence, is an expanding area of research that may have profound consequences for our understanding of the disease, and clinical care. There are various epigenetic changes that may occur as a direct result of TBI, including DNA methylation, histone modification, and changes in the levels of non-coding RNA. This review focuses on DNA methylation, its potential to alter the degree of injury, and the extent of recovery- including development of post-traumatic neurodegeneration, response to therapies, and the hereditable consequences of injury. The functional consequences of non-coding RNA and histone modifications are well described in the literature, but the mechanism by which these three mechanisms interact are often overlooked. Here, we briefly describe the interaction of DNA methylation with the two other key epigenetic changes, and highlight key work being done to understand the functional relevance of those mechanisms. The field of epigenetics is rapidly advancing due to the advent of less invasive and more versatile methods for measuring epigenetic proteins and their functional impact on cells, however, the evidence specific to TBI is limited. This review identifies several important outstanding questions that remain from the work already conducted, and highlights directions for the future. 


\section{The Effects of Traumatic Brain Injury on DNA Methylation}

\section{Traumatic Brain Injury and Epigenetics}

Traumatic Brain Injury (TBI) is heterogeneous disease that is a major cause of death and long-term disability. Over half of serious head trauma survivors may be moderately to severely disabled one-year post-injury, while long-term impairments resulting from mild repetitive TBI are increasingly being recognized. ${ }^{1}$ Thus, with incidence rates of over 100 per 100,000 people, TBI is an important public health concern and requires further exploration. ${ }^{2}$ Recent efforts to understand disease pathophysiology have focused on epigenetics, the process by which environmental factors alter gene expression without changing the underlying DNA sequence. Epigenetic changes are increasingly implicated in the pathophysiology following TBI (Figure 1), ${ }^{3}$ and may provide targets for therapy. This review seeks to provide an introduction epigenetics, and focus on specific aspects of the process as a context for understanding emerging publications on epigenetic changes in TBI.

\section{Epigenetics}

The DNA in nuclear chromatin is packaged into nucleosomes, which consist of 146 DNA base pairs wound around bundles of eight core histone proteins (often compared to a thread around a spool). In its inactive state, chromatin is tightly wound and compact, and as a consequence, DNA is not accessible for transcription. Modification of the nucleosome by a variety of processes selectively exposes segments of DNA (typically in promoter regions), and makes them available for transcription. Such selective transcription allows tissue and context specific expression of genes, despite a near-universal DNA sequence in all cells of a single individual.

Such modulation of gene expression can be effected either by modification of histone proteins (typically in exposed segments called tails), or by modifying DNA directly. Such modification, which collectively underlies epigenetic regulation, modifies nucleosome chemistry through variable methylation (either of DNA or histone tails; Box 1), or acetylation or phosphorylation (typically of histone tails). The exposure of segments of DNA (and hence accessibility their accessibility for transcription) can also be modulated by other factors such as non-coding RNA (ncRNA) segments 
(Box 2), which regulate histone modifying enzymes; or by remodelling complexes which slide DNA on the underlying histone spool, and make segments of DNA more or less accessible for transcription.

In TBI, these epigenetic processes could impact variability in injury susceptibility, the intensity of the pathophysiological response, response to therapies, the rate and extent of recovery, the development of post-traumatic neurodegeneration, and the hereditable consequences of injury. The following manuscript focuses on DNA methylation as a key epigenetic processes that is emerging as important in TBI pathophysiology:

\section{Figure 1}




\section{Box 1: DNA Methylation and Histone Modification - Two Sides of the Same Coin?}

The DNA in chromatin is packaged into nucleosomes, which consist of 146 DNA base pairs wound around bundles of eight (two units of $\mathrm{H} 2 \mathrm{~A}, \mathrm{H} 2 \mathrm{~B}, \mathrm{H} 3$ and $\mathrm{H} 4$ ) core histone proteins. Modification of the nucleosome by a variety of processes, allows selective accessibility of segments of DNA for transcription (typically in promoter regions), thus providing tissue and context specific expression of genes from a near-universal DNA sequence in all cells of a single individual. Such modifications can affect either the histone proteins (typically in exposed segments called tails), or by modifying the DNA directly.

Histone proteins can be covalently modified in at least 67 different ways, and the specific combination of these modifications determines the 'histone code'. ${ }^{4}$ Subsequently, this 'code' mediates specific protein-protein interactions contributing to short and long-term regulation of transcription, representing a form of cellular memory. ${ }^{5}$ This basic premise provides a mechanism by which alterations to histone proteins can result in epigenetic mechanism of gene regulation. When methyl binding proteins (MBPs) from the methyl-CpG binding domain (MBD) interact with methylated DNA sites in gene promoters they initiate a series of events, including recruitment of histone-modifying enzymes, deacetylation of histone protein tails, and chromatin condensation around the gene promoter. ${ }^{6}$ Consequently, the methylation of DNA results in the deacetylation of histones and as such there is a compounded inhibitory effect on transcription. ${ }^{7}$

Conversely, histone methylation can result in DNA methylation. For example, H3K27Me3, a trimethylated lysine residue at position 27 in histone $\mathrm{H} 3$, is associated with aberrant de novo DNA methylation. H3K27Me3 interacts with a DNA repression protein (the Polycomb complex), which, in association with another protein (EZH2), promotes DNMT-1 recruitment to the target chromatin complex, and results in DNA methylation. ${ }^{8}$ Direct evidence of changes in this complex interaction in TBI is still lacking and the functional importance is still unclear. One suggestion is that through this bidirectional crosstalk, epigenetic information from histone modifications is translated into a more stable record in the form of DNA methylation. 
Another hypothesis is that the direct interaction between MBPs and histone deacetylases, via a calciumdependent pathway, may represent one link between primary injury and late pathophysiology in TBI, at least in experimental models. However, there is also evidence to suggest that this calcium-mediated induction of specific genes may be entirely separate from the DNA methylation mechanism discussed thus far, and may actually be solely based on histone modifications. Both variants of the calciummediated epigenetic-modification based pathway for early gene induction are described in figure 2. 


\section{Box 2: DNA Methylation and Non-coding RNA}

Only $1-2 \%$ of the transcripts produced by genome transcription encode proteins. The functional consequences of the remaining noncoding ribonucleic acid (ncRNA) transcripts are poorly understood, yet they are associated with a variety of human diseases. ${ }^{15}$ The relationship between ncRNA and DNA methylation is best understood through the action of micro ribonucleic acids (miRNAs; small ncRNAs between 20-22 base pairs in length), which interact with specific messenger ribonucleic acids (mRNAs) to silence their expression post-transcriptionally. It is thought that some of these miRNAs downregulate the expression of DNMT, resulting in DNA hypomethylation. ${ }^{16}$

In one in vitro model of focal ischemia, miR-29c levels decreased two-fold after oxygen-glucose deprivation (OGD), relative to controls. However, pretreatment with pre-miR-29c increased postOGD level of miR-29c three-fold, and halved cell death. Therefore, in vitro, miR-29c appears to be a pro-survival factor, possibly via its inhibition of DNMT-3A, and its absence after ischemic injury seems to promote cell death. ${ }^{17}$ Recently miR-29 a, b, and c have been shown to be down-regulated after TBI. It is possible that the interactions between ncRNA and DNA methylation observed in models of ischemia, suggesting a pro-survival action for MiR-29c, could also be true of TBI, however this proposition requires additional investigation. ${ }^{18}$

Alternatively, the functional consequence of altered microRNA expression may be mediated via changes to mitochondrial function. Support for this proposition comes from the demonstration that microRNAs can regulate aspects of mitochondrial function (including mitochondrial gene expression associated with apoptosis and oxidative stress responses), ${ }^{19}$ and the data that mitochondrial function in TBI models can be modulated by changes in miRNA levels, as shown by Wang et al. ${ }^{20}$ As such, a growing area of interest is DNA methylation at the level of mitochondrial DNA, and how we might be able to produce novel therapies via modulation of the mitochondrial DNA methyltransferase enzymes. ${ }^{21,22}$ 


\section{Mechanisms of DNA methylation and demethylation}

The process of DNA methylation occurs predominantly at cysteine bases, which are converted to 5methylcytosine. In mammals, methylation targets cytosine bases that exist as part of a CpG (cytidinephosphate-guanidine) dinucleotide. ${ }^{23}$ Long stretches of DNA that are densely populated by CpGs are described as CpG islands (CpGIs), and are most commonly found in the proximal promoter region of genes. ${ }^{24}$ These genes are often associated with house-keeping functions including gene expression mechanisms, DNA replication in the nucleus, metabolism, and cell cycle control. ${ }^{7}$ The role of nonCpG associated methylation is still controversial, and its functional consequences are poorly understood. ${ }^{25}$ In $\mathrm{CpG}$ islands, DNA methylation directly prevents the necessary factor and regulatory units from accessing the gene promoter ${ }^{26}$ thereby inhibiting gene transcription. The process of DNA methylation and de-methylation are carried out by specific enzymes, the activity of which has been shown to change in experimental models of neural injury.

\section{DNA Methyltransferases (DNMT-1/3A/3B)}

The DNMT family of enzymes carry out the process of DNA methylation. DNMT-3B is predominantly expressed in embryonic and neural precursor cells while DNMT-3A is principally expressed later in development, in maturing neurons, oligodendrocytes, and astrocytes. ${ }^{27,28}$ DNMT-1 is expressed in post-mitotic CNS neurons, and serves to institute and preserve the high local levels of DNA methylation. ${ }^{21}$ Essentially, DNMT-3A/B can be thought of as the de novo methyltransferases, whilst DNMT-1 maintains methylation status.

\section{Ten-eleven Translocation Metbylcytosine Dioxygenase}

The ten-eleven translocation methylcytosine dioxygenase (TET) family of proteins has been proposed as a viable mechanism for active demethylation. ${ }^{29}$ Bailey and colleagues demonstrated that exposure to simulated free-field blast exposure, at 10 and 23 PSI resulted in significantly increased TET2, but not TET1 expression in the prefrontal cortices and hippocampi of male Sprague Dawley rats. ${ }^{30}$ Moreover, these changes were correlated with incrementally greater levels of global hypomethylation compared to shams. However, three recent loss-of-function mutation experiments demonstrate incongruent results regarding the effects of TET2 on DNA methylation status. ${ }^{31-33}$ An alternative explanation, is 
that TET2 could be preventing protracted inflammation by suppressing macrophage IL- 6 expression, via the recruitment of histone deacetylase 2 (HDAC2) and histone deacetylation (a DNA methylation independent mechanism). ${ }^{34}$ Therefore, the increased inflammation associated with higher blast pressures also explains the observed increase in TET2 expression.

Growth arrest and DNA-damage-inducible protein (GADD45)

GADD45 has been identified as a mediator of active DNA demethylation. ${ }^{35}$ The potential relevance of such this process to neurotrauma can be inferred from demonstration that peripheral nerve injury decreases the repressive methylation of the GADD45a promoter. ${ }^{36}$ Intriguingly, systemic folate therapy, in a model of combined spinal and nerve injury, increases methylation of the GADD45a promoter at 12 of its $18 \mathrm{CpG}$ sites, restores its methylation levels to baseline, and promotes spinal regeneration. ${ }^{37}$ Based on these findings, it would be of great interest to see whether GADD45 might also play a role in active demethylation in experimental models of TBI.

DNA Methyltransferases in TBI: Temporal, regional, and cellular patterns and functional implications

Whilst the action of the DNA methyltransferases is relatively well understood in non-pathological circumstances, the impact and action of these enzymes in pathological circumstances is not well understood. The following discussion addresses some issues that are of direct or indirect relevance to TBI.

\section{DNA methylation changes in acute TBI}

DMNT-1 expression is upregulated following a controlled cortical impact injury (CCI) in the prefrontal cortices and hippocampi of both male and female Sprague Dawley pups (21 days old at trauma, 47 at sacrifice). ${ }^{38}$ However, these brain areas have several different roles, and cell types, making it impossible to infer the functional relevance of this upregulation, especially given the evidence (discussed later) that genes are differentially affected, and can display either hyper- or hypo-methylation post-injury. ${ }^{39}$

Moreover, the global increase described by the previous study may be misleading, as more targeted analysis of DMNT-1 in pathological and non-pathological circumstances shows specific cellular and 
intra-cellular patterns of expression. Lundberg et al showed that DMNT-1 expression in normal rat cortex, is almost exclusively nuclear. ${ }^{40}$ However, some peri-ventricular cells (in both injured and uninjured rat brain) co-express nestin and DNMT1 in both nucleus and cytoplasm. ${ }^{40}$ Given that nestin, an intermediate filament protein, is a histological marker of neuronal precursor cells, ${ }^{41}$ its colocalisation with cytoplasmic DNMT-1 suggests that the enzyme may play a role in neuronal differentiation.

\section{Cellular specificity of changes in DNA methylation}

Subsequently, Lundberg and colleagues described the impact of TBI on the expression of DMNT-1. At four days post-CCI in male Sprague Dawley rats, peri-lesional nestin, glial fibrillary astrocytic protein (GFAP), and neuronal nuclei (NeuN) stained cells demonstrated both nucleic and cytoplasmic localization of DNMT-1.40 GFAP is a known histological marker for astrocytes. ${ }^{41}$ There was no corresponding cytoplasmic localization of DNMT-1 in microglial cells. The quantity of cytoplasmic DNMT-1 declined at each subsequent time point, and was almost completely absent by 14 days postinjury. ${ }^{40}$ Based on the illustration of both a spatial (nuclear to cytoplasmic shift) and temporal (cytoplasmic immunoreactivity absence by day 14) relationship between TBI and DNMT-1, it may be that a similar process to physiological differentiation is induced in peri-lesional reactive astrocytes postinjury. ${ }^{40}$ Assuming that the presence of nestin implies that the cells have transitioned to a less differentiated state, the JAK/STAT pathway is may provide a plausible link in this process. DNA methylation status is thought to control the timing of astrocyte differentiation, through inhibition of STAT activity. Moreover, in the developing CNS, global hypomethylation it thought to promote active chromatin remodelling and expression of astroglial marker genes, manifesting as precocious astroglial differentiation. ${ }^{42}$ Thus, Lundberg and colleagues argue, DNMT1 re-localization may induce expression of specific genes in the JAK-STAT pathway that are critical in astrocyte differentiation, ${ }^{40}$ specifically through increased JAK-STAT signalling, as well as demethylation of the STAT binding element within the GFAP promoter. ${ }^{42}$ Therefore, DNMT1 re-localization could contribute to the epigenetic reprogramming of in situ reactive astrocytes, as a result of the brain trauma, explaining the persistence of astrogliosis. ${ }^{42}$

Since DNA methylation is important in the regulation of cell-specific gene transcription, it is 
unsurprising that neurons and glia demonstrate differing methylation patterns under basal condition. ${ }^{43}$ In a blast-exposure model (equal to 10.8 PSI), Haghighi and colleagues identified 1807 cytosine sites where hyper- and hypo-methylation followed 8 months post TBI, in the prefrontal cortex of 10 weekold male Long Evans hooded rats. They demonstrated that methylation status varied in a wide variety of different genes in neurons and glia. ${ }^{39}$ These sites corresponded to 837 genes in the rat genome, of which 458 were specific to proteins found exclusively in neurons and are associated with cell death, growth, proliferation, and nervous system development. The remaining 379 altered genes were specific to proteins found exclusively in glia, and are associated with: enrichment in cancer, cellular growth and proliferation, as well as nervous system development and function. Notably, 92 of affected genes have been previously implicated in neurological disease pathogenesis, and 39 in neuropsychiatric disorders. The only other study directly examining DNA methylation following TBI found, in an otherwise normal rat brain library, evidence that the hypomethylation was localized to a subpopulation of reactive microglia. ${ }^{44}$ The latter study utilized a weight-drop contusion model in 8-9 week-old Lewis rats, and examined contused somatosensory cortex at $24,48,96$ or 144 hours after brain injury.

Whilst apparently incongruous, the differences in results from these studies may be attributed to differences in brain region examined (pre-frontal vs. somatosensory cortex), traumatic brain injury model (simulated blast vs. weight drop contusion), and time from exposure to analysis (hours/days vs. 8 months). Further, methodological factors may account for some of the differences observed. One of the limitations of simple bisulfite sequencing is that the process is unable to distinguish between 5methylcytosine (the target), and 5-hydroxymethylcytosine: the product of a TET protein mediated reaction. ${ }^{45}$ TET2 2 is thought to be important in resolving inflammation by suppressing IL- $6,{ }^{34}$ which is known to be produced by macrophages/microglia. It is plausible that the significant increase in qualitatively observed methylation in the Zhang study is due to the inclusion of 5hydroxymethylcytosine labelling, ${ }^{44}$ which fits with the localization in the microglial sub-population. This is especially convincing when we consider that the administration of dexamethasone, an antiinflammatory agent, led to the reduced levels of the hypomethylation, in a dose-dependent manner. ${ }^{44}$ Reduced inflammation would mean less TET2 is required to resolve the inflammatory response, and therefore there would be fewer TET protein mediated alterations to the DNA. The Haghighi study 
utilized a specific variant of the TET assisted bisulfite sequencing method developed by Yu et al. capable of labelling just the intended target. ${ }^{39,46,47} \mathrm{Had}$ the updated form of bisulphite sequencing not been utilized, they might have produced similar results to Zhang et al., ${ }^{44}$ as the modifications by the TET proteins would have resulted in an artificially high level of DNA methylation in the microglial DNA profile.

\section{$D N A$ Methylation and TGF- $\beta$}

Epigenetic methylation causes interesting changes in the biological pathway of transforming growth factor $\beta$ (TGF- $\beta$ ), a cytokine that demonstrates significantly increased activity in neuronal cells, but not in glia, after blast-related TBI (see Haghighi et al., 2015). ${ }^{39}$ A recent cortical controlled impact model study using 8 week old C57BL/6 mice also demonstrated increased activity in this pathway, 1,3, and 7 days post-traumatic injury, specifically in the forebrain subventricular zone and the hippocampal dentate gyrus, brain areas associated with neurogenesis. ${ }^{48}$ Normally, TGF- $\beta$ signaling has a role in cell proliferation, synaptic transmission and adult neurogenesis. ${ }^{49,50}$ The functional consequences of upregulation of the TGF- $\beta$ pathway is currently under investigation. ${ }^{51,52}$ However, it remains unclear whether upregulation of this pathway post-injury represents a part of the recovery process, or if TGF$\beta_{1}$ contributes to CNS scar formation, ${ }^{53}$ prevents regeneration, and contributes to late pathophysiology. The interaction of TGF- $\beta_{1}$ and the JAK-STAT pathway in promoting astrogliosis is recognized, ${ }^{54}$ but no specific data are available in the context of TBI.

\section{DNA Methylation and Neurodegeneration}

Epidemiological studies suggest that TBI may be an important epigenetic risk factor for Alzheimer's disease (AD), although this remains a contentious subject, especially with regards to the mild TBI population. ${ }^{55}$ Two examples suggest that DNA methylation might elucidate the pathobiology behind this epidemiological link. Firstly, evidence from a rodent study (12-14 month old female SpragueDawley rats ${ }^{56}$ suggests that TGF- $\beta$, which is thought to be increased post-traumatic injury, 39,48 increases expression of amyloid precursor protein (APP) and deposition of beta amyloid (A $\beta$ ), both of which are implicated in the pathogenesis of Alzheimer's disease. ${ }^{56}$ Another potential link between TBI and $\mathrm{AD}$ is based on recent in-vitro descriptions of DNA methylation in microglia cell lines as an 
important influence on the expression of genes associated with Alzheimer's disease pathology, including beta secretase 1 (BACE1) and Presenilin 1.57-59 Both hypotheses are relatively nascent and require further research to demonstrate how the effect achieved following the primary insult is sufficiently long lived to the produce the delayed presentation of dementia.

\section{A potential role in post-traumatic epilepsy: Insights from ischemic brain injury}

Although TBI and ischaemia are not identical, ${ }^{60}$ neuronal responses in ischaemia provide additional mechanistic insights into the potential role of DNA methylation in TBI. For example, an ischemiareperfusion injury (IRI) in gerbils results in rapid reductions in DNMT1 activity in pyramidal (but not non-pyramidal) cells. Five days post-IRI DNMT1 immunoreactivity was localized to GABAergic interneurons, and astrocytes, but not microglia, ${ }^{61}$ a finding concordant with some of the data in TBI, and suggesting that glial cells can undergo epigenetic modification in response to, or as a result of injury. Furthermore, the specificity of expression in GABAergic neurons suggests potential switching from from the normal post-natal expression of the type 2 inwardly directed $\mathrm{Na}^{+} / \mathrm{K}^{+} / 2 \mathrm{Cl}^{-}$cotransporter (NKCC2), ${ }^{62}$ to the type 1 cotransporter (NKCC1; which is more readily epigenetically modulated, ${ }^{63}$ preferentially expressed during prenatal development, and results in a depolarizing response). ${ }^{64}$ Indeed, axotomy, ischemia and excitotoxicity, all of which mechanisms are recognized in TBI, are associated with changes in intracellular $\mathrm{Cl}^{-}$concentrations thought to be caused by a reversion to predominantly NKCC1 expression.65,66 DNMT1 expression in GABAergic interneurons following neural injury could thus change neuronal excitability, and link primary and secondary injury in TBI. Indeed, Wang et al have shown that NKCC1 upregulation appears to mediate post-traumatic seizures, and that inhibition of this pathway by genetic deletion or pharmacological inhibition suppresses TBIinduced seizures. ${ }^{67}$ Furthermore, this seizure pathway may be additionally driven by TGF- $\beta$ expression, suggesting a critical nexus for epigenetic mechanisms associated with TBI.

\section{DNA Methylation in circadian rbythms and sleep physiology}

Common symptoms following TBI include sleep disturbances and depression. ${ }^{68}$ Sleep deprivation may be related to reduced melatonin levels observed post-TBI and contribute to impaired psychiatric and neurological recovery. ${ }^{69}$ In a blast-model of TBI, expression of the Aanat gene was modestly 
decreased as a result of increased methylation. ${ }^{39}$ The Aanat gene encodes an enzyme (Aralkylamine $\mathrm{N}$-acetyltransferase) involved in the conversion of serotonin to melatonin, which is important in entrainment of the circadian rhythms, and both the gene and the pathway have also been linked to depression susceptibility.70,71 TBI-specific epigenetic changes, such as Aanat suppression, might disrupt the normal circadian patterns explaining why symptoms such as sleep disruption are associated with TBI. Light induced histone phosphorylation is also known to regulate the function of the central circadian pacemaker, the suprachiasmatic nucleus through activation of immediate early genes including BDNF.72 Furthermore, such histone modifications can alter rhythmic activity in other organs in a manner similar to that of imprinted pathologies. ${ }^{73}$ Further exploration of these links between TBI and epigenetics could elucidate pathophysiology and identify potential new therapeutic targets in TBI.

\section{Clinical applications: therapy and prognostication}

While there are no current therapies for TBI that target epigenetic pathways, several potential examples suggest pathways for such development.

Lithium shows several potentially beneficial effects in TBI including the induction of autophagy and the ability to prevent excess inflammation by inhibiting microglia activation. ${ }^{74}$ However, lithium may also modulate epigenetic mechanisms in the brain, as suggested by its role bipolar disorder (BD), where it may restore or increase the levels of BDNF and so reduce the repressive hypermethylation of the BDNF promoter region. ${ }^{75} \mathrm{~A}$ similar mechanism might be activated with lithium treatment post-TBI. The DNA methylation inhibitor, zebularine, which proved capable of reducing the extent injury in an ischemia model, ${ }^{76}$ corroborating findings that a partial genetic deletion of DNMT-1 enzyme improved outcome in experimental stroke. ${ }^{77}$

Folic acid is a methyl donor substrate, and a vital ingredient in the production of cellular S-adenosylmethionine, which in turn is required by DNMTs. Given that folate administration can repress the demethylating action of growth arrest and DNA damage-inducible gene 45 alpha (GADD45), ${ }^{36}$ and its ability to suppress DNMT-3A/3B, but not DNMT-1, and thereby restore baseline methylation status, 
folate may have a therapeutic role post TBI. ${ }^{37}$ Evidence for the efficacy of folate as a pharmacological intervention can be seen in a recent piglet model of pediatric TBI, which found folate supplementation led to significantly improved functional recovery as compared to controls at day one post head injury (although these gains did not persist to day four)..$^{78}$ Additional evidence is needed, but the safety of folate supplementation makes this an attractive early target for a clinical trial, with an explanatory arm that demonstrates the mechanistic basis for any benefit seen.

A better understanding of which genes undergo epigenetic changes, how this alters their function, and the relationship to TBI pathology, may allow development of a prognostic stratification based on these epigenetic modifications. ${ }^{79}$ Whilst we have highlighted several potential candidates linking epigenetic alterations to TBI pathophysiology, our current understanding of individual variation post-TBI remains insufficient to determine whether these factors might be of prognostic significance.

Finally, much of the discussion thus far has focused on the changes that occur downstream of the DNA methylation. However, understanding the biochemical process that precedes the epigenetic modifications, may allow us to identify potential interventions to prevent or modify them, before they can become detrimental to the individual's health. The evidence available suggests that calcium plays a pivotal role in linking trauma to the epigenetic changes described previously. Figure 2 illustrates how calcium related change that occur due to trauma can lead to both DNA methylation and histone modifications.

\section{Practical Considerations}

\section{Extrapolations from other pathologies: a cautionary note}

Other publications, and earlier sections of this manuscript, have drawn on knowledge of physiological mechanisms of epigenetic modulation, and its alteration in diseases other than TBI, to provide a basis for further studies. While there may be substantial commonalities amongst diseases, it is important to recognize potential discordances. Debski and colleagues investigated DNA methylation patters in three models of acquired epilepsy, of which one was TBI, and found that they did not share any evidence of a common epigenetic signature, but instead appeared to suggest that different epileptogenic (biological) 
processes prevailed in each model. ${ }^{80}$ As such, it is of paramount importance that we exercise caution in transferring epigenetic models of pathobiology from other diseases to TBI.

\section{Confounding Factors}

Recruitment bias in epigenome wide association studies (EWAS) is a key issue for future studies. ${ }^{81}$ Particular confounds may arise from socioeconomic/environmental effects on DMNT-1 expression. Calorie restriction and dietary fat content have been incidentally noted to have significant effects on DMNT-1 expression, which vary with sex in animal models. ${ }^{82}$ It would be important to explore similar effects in humans TBI, particularly given the recent demonstrations of diet-dependent (and potentially heritable) epigenetic changes. ${ }^{83}$ If confirmed, this phenomenon has important implications for sample size, recording of socioeconomic and dietary history, and the use of best practice statistical methods such as multiple testing corrections and mitigation of batch effects. ${ }^{81}$

\section{Temporality}

The reversible and therefore dynamic nature of epigenetic changes adds an additional layer of complexity to describing the changes that occur in response to pathology, since the temporal context (e.g. time since trauma) in which those changes have occurred is of vital importance. Point estimates of epigenetic variation/alteration fail to capture that additional complexity, and thus, could lead to spurious conclusions about the role of epigenetic modifications. Only very recently have studies begun to characterize the temporal profiles of these changes. ${ }^{84}$

\section{Routes to human translation}

Access to brain tissue: One of the key obstacles in epigenetic research, in the context of brain injury, is the difficulty in securing viable tissues samples in living individuals for sequencing. In the context of ethically approved research, it may be possible to harvest cerebrospinal fluid, or brain tissue during routine clinical care for epigenetic analysis. However, such sampling is biased to either the early period after TBI, or to terminal pathophysiology in autopsy specimens. Control (i.e. non-TBI) tissue may,

paradoxically, be also more difficult to access in this context. Tissue from spontaneously aborted or terminated pregnancies, ${ }^{85}$ can provide insight into DNA methylation during the early stages of human 
development and even the impact of specific types of pathology, like ischemia, on the epigenome, but cannot strictly be used as control samples for TBI studies.

Imaging epigenetic changes in vivo: Positron emission tomography (PET) using $\left[{ }^{11} \mathrm{C}\right]$ Martinostat, an imaging probe selective for specific classes of histone deacetylases (HDACs) most associated with regulating neuroplasticity, has been described, and allows in vivo quantification of HDAC activity in vivo in humans. ${ }^{86}$ Although this technology is still young, and therefore can only be used to answer very specific hypothesize, it represents a trend toward non-invasive measurement of epigenetic modifications which are important given the constantly fluctuating nature of the changes. ${ }^{84}$

Circulating DNA analysis: Another non-invasive method that has become increasingly relevant in the last 5 years is the analysis of cell free circulating DNA. Examination of both serum and CSF samples for DNA methylation patterns have been found to be useful in identifying tissue specific cell death. A greater understanding of brain cell type specific methylation patterns may allow for even more specific monitoring beyond the authors description of gross measurement of brain cell death post-traumatic brain injury. ${ }^{87}$ However, it should be noted that the potential usefulness of this method is still a matter of debate. Using unsupervised clustering and principal component analysis of eye tissue and peripheral blood sample epigenetic profiles, Hewitt and colleagues demonstrate that the origin of the tissue appears to be the main driver of variation in methylation patterns. ${ }^{88}$ Moreover, there was only limited covariation between the blood and specific eye tissues. Conversely, other studies have demonstrated that when a specific gene is known to have functional/diagnostic/treatment-related relevance it may be possible to too look for promotor methylation changes in such peripherally obtained samples, as has been done with serum and CSF probes for MGMT promotor methylation in glioma patients. ${ }^{89}$

Assessing epigenetic changes in the host response after TBI: While the discussions above have focused on assessment of epigenetic changes in the brain, it is important to recognize that TBI results in a substantial host response which involves both innate and adaptive immunity..$^{90}$ As in many other diseases, these changes may be epigenetically driven, and potentially contribute to both early and late pathophysiology following TBI. Examination of epigenetic changes in peripheral blood mononuclear 
cells (PBMCs) can be confounded by changes in cell populations, but if these can be mitigated by cell sorting, epigenomic analysis of peripheral immune cells may provide a tractable, and potentially important tool in understanding the late consequences of TBI.91,92

\section{Conclusion}

The role of epigenetic changes, and particularly DNA methylation, in TBI-related pathology is increasingly being explored. The experimental literature has identified many epigenetic changes which in turn lead to changes in neuronal and glial function. For example, changes in DNMT-1 immunoreactivity altered methylation status of glia and neurons, DNA methylation and transcription of noncoding RNA. These epigenetic alterations may provide a mechanism for longer-term changes and even hereditable consequences. However, the mechanisms of these processes as well as the impacts of different injury patterns and individual variation on epigenetic response are not yet understood fully. There is a strong case for further work that aims to explain the basic pathobiology of epigenetic modification in TBI, rather than epigenome level associative studies, as a keen understanding of the former is necessary to interpret the results of the latter. Key outstanding questions are presented in table 1. The full extent of how epigenetic change contributes to or mitigates secondary brain injury remains unclear, and is an important area of future research. 


\section{Author Disclosure Statement}

The authors have no competing financial interests. This study received no funding. 


\section{$\underline{\text { References }}$}

1. Thornhill, S., Teasdale, G. M., Murray, G. D., McEwen, J., Roy, C. W., and Penny, K. I. (2000). Disability in young people and adults one year after head injury: prospective cohort study. BMJ 320, 1631-1635.

2. Huang, Y., Pastor, W. A., Shen, Y., Tahiliani, M., Liu, D. R., and Rao, A. (2010). The behaviour of 5-hydroxymethylcytosine in bisulfite sequencing. PloS one 5, e8888.

3. Waddington, C. H. (2012). The epigenotype. Int. J. Epidemiol. 41, 10-13.

4. Tan, M., Luo, H., Lee, S., Jin, F., Yang, J.S., Montellier, E., Buchou, T., Cheng, Z., Rousseaux, S., Rajagopal, N. and Lu, Z. (2011). Identification of 67 histone marks and histone lysine crotonylation as a new type of histone modification. Cell 146, 1016-1028.

5. Maze, I., Wenderski, W., Noh, K. M., Bagot, R. C., Tzavaras, N., Purushothaman, I., Elsässer, S. J., Guo, Y., Ionete, C., Hurd, Y. L. and Tamminga, C. A. (2015). Critical role of histone turnover in neuronal transcription and plasticity. Neuron 87, 77-94.

6. Baylin, S. B. and Jones, P. A. (2011). A decade of exploring the cancer epigenome-biological and translational implications. Nat. Rev. Cancer 11, 726-734.

7. Siegfried, Z. and Simon, I. (2010). DNA methylation and gene expression. Wiley Interdiscip. Rev. Syst. Biol. Med. 2, 362-371.

8. Viré, E., Brenner, C., Deplus, R., Blanchon, L., Fraga, M., Didelot, C., Morey, L., Van Eynde, A., Bernard, D., Vanderwinden, J.M. and Bollen, M. (2006). The Polycomb group protein EZH2 directly controls DNA methylation. Nature 439, 871-874.

9. Lee, H. A., Hong, S. H., Kim, J. W. and Jang, I. S. (2010). Possible involvement of DNA methylation in NKCC1 gene expression during postnatal development and in response to ischemia. Journal of neurochemistry 114, 520-529.

10. Wang, F., Wang, X., Shapiro, L. A., Cotrina, M. L., Liu, W., Wang, E. W., Gu, S., Wang, W., He, X., Nedergaard, M. and Huang, J. H. (2016). NKCC1 up-regulation contributes to early posttraumatic seizures and increased post-traumatic seizure susceptibility. Brain Structure and Function 1, 1-14.

11. Leeds, P. R., Yu, F., Wang, Z., Chiu, C. T., Zhang, Y., Leng, Y., Linares, G. R. and Chuang, D. M. (2014). A new avenue for lithium: intervention in traumatic brain injury. ACS Chem. Neurosci. 5, 422-433. 
12. D'addario, C., Dell'Osso, B., Palazzo, M.C., Benatti, B., Lietti, L., Cattaneo, E., Galimberti, D., Fenoglio, C., Cortini, F., Scarpini, E. and Arosio, B. (2012). Selective DNA methylation of BDNF promoter in bipolar disorder: differences among patients with BDI and BDII. Neuropsychopharmacology 37, 1647-1655.

13. Endres, M., Meisel, A., Biniszkiewicz, D., Namura, S., Prass, K., Ruscher, K., Lipski, A., Jaenisch, R., Moskowitz, M. A. and Dirnagl, U. (2000). DNA methyltransferase contributes to delayed ischemic brain injury. J. Neurosci. 20, 3175-3181.

14. Dock, H., Theodorsson, A. and Theodorsson, E. (2015). DNA methylation inhibitor zebularine confers stroke protection in ischemic rats. Transl. Stroke Res. 6, 296-300.

15. Lo, R. and Weksberg, R. (2014). Biological and biochemical modulation of DNA methylation. Epigenomics 6, 593-602

16. Chen, B. F., Gu, S., Suen, Y. K., Li, L. and Chan, W. Y. (2014). microRNA-199a-3p, DNMT3A, and aberrant DNA methylation in testicular cancer. Epigenetics, 9, 119-128.

17. Pandi, G., Nakka, V. P., Dharap, A., Roopra, A. and Vemuganti, R. (2013). MicroRNA miR-29c down-regulation leading to de-repression of its target DNA methyltransferase 3a promotes ischemic brain damage. PLoS One 8, p.e58039.

18. Miao, W., Bao, T. H., Han, J. H., Yin, M., Yan, Y., Wang, W. W. and Zhu, Y. H. (2015). Voluntary exercise prior to traumatic brain injury alters miRNA expression in the injured mouse cerebral cortex. Braz. J. Med. Biol. Res. 48, 433-439.

19. Li, P., Jiao, J., Gao, G. and Prabhakar, B. S. (2012). Control of mitochondrial activity by miRNAs. J. Cell. Biochem. 113, 1104-1110.

20. Wang, W. X., Sullivan, P. G. and Springer, J. E. (2017). Mitochondria and microRNA crosstalk in traumatic brain injury. Prog. Neuropsychopharmacol. Biol. Psychiatry 73, 104-108.

21. Chestnut, B. A., Chang, Q., Price, A., Lesuisse, C., Wong, M. and Martin, L. J. (2011). Epigenetic regulation of motor neuron cell death through DNA methylation. J. Neurosci. 31, 661916636.

22. Shock, L. S., Thakkar, P. V., Peterson, E. J., Moran, R. G. and Taylor, S. M. (2011). DNA methyltransferase 1 , cytosine methylation, and cytosine hydroxymethylation in mammalian mitochondria. Proc. Natl. Acad. Sci. USA 108, 3630-3635.

23. Law, J. A. and Jacobsen, S. E. (2010). Establishing, maintaining and modifying DNA methylation patterns in plants and animals. Nat. Rev. Genet. 11, 204-220. 
24. Bird, A. (2002). DNA methylation patterns and epigenetic memory. Genes Dev. 16, 6-21.

25. He, Y. and Ecker, J. R. (2015). Non-CG methylation in the human genome. Annu. Rev. Genom. Hum. Genet. 16, 55-77.

26. Watt, F. and Molloy, P. L. (1988). Cytosine methylation prevents binding to DNA of a HeLa cell transcription factor required for optimal expression of the adenovirus major late promoter. Genes Dev. 2,1136-1143.

27. Okano, M., Bell, D. W., Haber, D. A. and Li, E. (1999). DNA methyltransferases Dnmt3a and Dnmt3b are essential for de novo methylation and mammalian development. Cell 99, 247257.

28. Watanabe, D., Uchiyama, K. and Hanaoka, K. (2006). Transition of mouse de novo methyltransferases expression from Dnmt3b to Dnmt3a during neural progenitor cell development. Neuroscience 142, 727-737.

29. Karuppagounder, S.S., Kumar, A., Shao, D.S., Zille, M., Bourassa, M.W., Caulfield, J.T., Alim, I. and Ratan, R.R. (2015). Metabolism and epigenetics in the nervous system: Creating cellular fitness and resistance to neuronal death in neurological conditions via modulation of oxygen-, iron-, and 2-oxoglutarate-dependent dioxygenases. Brain Res. 1628, 273-287.

30. Bailey, Z. S., Grinter, M. B., Campos, D. D. L. T. and VandeVord, P. J. (2015). Blast induced neurotrauma causes overpressure dependent changes to the DNA methylation equilibrium. Neurosci. Lett. 604, 119-123.

31. Figueroa, M. E., Abdel-Wahab, O., Lu, C., Ward, P. S., Patel, J., Shih, A., Li, Y., Bhagwat, N., Vasanthakumar, A., Fernandez, H. F. and Tallman, M. S. (2010). Leukemic IDH1 and IDH2 mutations result in a hypermethylation phenotype, disrupt TET2 function, and impair hematopoietic differentiation. Cancer cell 18, 553-567.

32. Ichiyama, K., Chen, T., Wang, X., Yan, X., Kim, B. S., Tanaka, S., Ndiaye-Lobry, D., Deng, Y., Zou, Y., Zheng, P. and Tian, Q. (2015). The methylcytosine dioxygenase Tet2 promotes DNA demethylation and activation of cytokine gene expression in T cells. Immunity 42, 613-626.

33. Ko, M., Huang, Y., Jankowska, A. M., Pape, U. J., Tahiliani, M., Bandukwala, H. S., An, J., Lamperti, E. D., Koh, K. P., Ganetzky, R. and Liu, X. S. (2010). Impaired hydroxylation of 5methylcytosine in myeloid cancers with mutant TET2. Nature 468, 839-843.

34. Zhang, Q., Zhao, K., Shen, Q., Han, Y., Gu, Y., Li, X., Zhao, D., Liu, Y., Wang, C., Zhang, X. and Su, X. (2015). Tet2 is required to resolve inflammation by recruiting Hdac2 to specifically repress 
IL-6. Nature 525, 389-393

35. Barreto, G., Schäfer, A., Marhold, J., Stach, D., Swaminathan, S. K., Handa, V., Döderlein, G., Maltry, N., Wu, W., Lyko, F. and Niehrs, C. (2007). Gadd45a promotes epigenetic gene activation by repair-mediated DNA demethylation. Nature 445, 671-675.

36. Befort, K., Karchewski, L., Lanoue, C. and Woolf, C.J. (2003). Selective up-regulation of the growth arrest DNA damage-inducible gene Gadd45 alpha in sensory and motor neurons after peripheral nerve injury. European Journal of Neuroscience 18, 911-922.

37. Iskandar, B., Rizk, E., Meier, B., Hariharan, N., Bottiglieri, T., Finnell, R., Jarrard, D., Banerjee, R., Skene, J., Nelson, A., Patel, N., Gherasim, C., Simon, K., Cook, T. and Hogan, K. (2010). Folate regulation of axonal regeneration in the rodent central nervous system through DNA methylation. Journal of Clinical Investigation 120, 1603-1616.

38. Mychasiuk, R., Hehar, H., Ma, I. and Esser, M. J. (2015). Dietary intake alters behavioral recovery and gene expression profiles in the brain of juvenile rats that have experienced a concussion. Front. Behav. Neurosci. 9, 17

39. Haghighi, F., Ge, Y., Chen, S., Xin, Y., Umali, M.U., De Gasperi, R., Gama Sosa, M. A., Ahlers, S. T. and Elder, G. A. (2015). Neuronal DNA methylation profiling of blast-related traumatic brain injury. J. Neurotrauma 32, 1200-1209.

40. Lundberg, J., Karimi, M., von Gertten, C., Holmin, S., Ekström, T. J. and Sandberg-Nordqvist, A. C. (2009). Traumatic brain injury induces relocalization of DNA-methyltransferase 1. Neurosci. Lett. 457, 8-11.

41. Holmin, S., von Gertten, C., Sandberg-Nordqvist, A. C., Lendahl, U. and Mathiesen, T. (2001). Induction of astrocytic nestin expression by depolarization in rats. Neurosci. Lett. 314, 151155.

42. Fan, G., Martinowich, K., Chin, M. H., He, F., Fouse, S. D., Hutnick, L., Hattori, D., Ge, W., Shen, Y., Wu, H. and ten Hoeve, J. (2005). DNA methylation controls the timing of astrogliogenesis through regulation of JAK-STAT signaling. Development 132, 3345-3356.

43. Iwamoto, K., Bundo, M., Ueda, J., Oldham, M.C., Ukai, W., Hashimoto, E., Saito, T., Geschwind, D. H. and Kato, T. (2011). Neurons show distinctive DNA methylation profile and higher interindividual variations compared with non-neurons. Genome Res. 21, 688-696.

44. Zhang, Z. Y., Zhang, Z., Fauser, U. and Schluesener, H. J. (2007). Global hypomethylation defines a sub-population of reactive microglia/macrophages in experimental traumatic brain 
injury. Neurosci. Lett. 429, 1-6.

45. Huang, Y., Pastor, W. A., Shen, Y., Tahiliani, M., Liu, D. R., and Rao, A. (2010). The behaviour of 5-hydroxymethylcytosine in bisulfite sequencing. PloS one 5(1), e8888.

46. Garrett-Bakelman, F. E., Sheridan, C. K., Kacmarczyk, T. J., Ishii, J., Betel, D., Alonso, A., ... \& Melnick, A. M. (2015). Enhanced reduced representation bisulfite sequencing for assessment of DNA methylation at base pair resolution. JoVE (Journal of Visualized Experiments), (96), e52246-e52246.

47. Yu, M., Hon, G. C., Szulwach, K. E., Song, C. X., Jin, P., Ren, B. and He, C. (2012). Tet-assisted bisulfite sequencing of 5-hydroxymethylcytosine. Nat. Protoc. 7(12), 2159-2170.

48. Logan, T. T., Villapol, S. and Symes, A. J. (2013). TGF- $\beta$ superfamily gene expression and induction of the Runx1 transcription factor in adult neurogenic regions after brain injury. PloS one 8, p.e59250.

49. Horbelt, D., Denkis, A. and Knaus, P. (2012). A portrait of Transforming Growth Factor $\beta$ superfamily signalling: Background matters. Int. J. Biochem. Cell Biol. 44, 469-474.

50. Krieglstein, K., Zheng, F., Unsicker, K. and Alzheimer, C. (2011). More than being protective: functional roles for TGF- $\beta$ /activin signaling pathways at central synapses. Trends Neurosci. 34, 421-429.

51. Chio, C. C., Lin, M. T., Chang, C. P., \& Lin, H. J. (2016). A positive correlation exists between neurotrauma and TGF- $\beta 1$-containing microglia in rats. European journal of clinical investigation, 46(12), 1063-1069.

52. Wang, X. Y., Ba, Y. C., Xiong, L. L., Li, X. L., Zou, Y., Zhu, Y. C., ... \& Li, J. T. (2015). Endogenous TGFß1 Plays a Crucial Role in Functional Recovery After Traumatic Brain Injury Associated with Smad3 Signal in Rats. Neurochemical research, 40(8), 1671-1680.

53. Logan, A., Berry, M., Gonzalez, A. M., Frautschy, S. A., Sporn, M. B. and Baird, A. (1994). Effects of transforming growth factor $\beta 1$, on scar production in the injured central nervous system of the rat. Eur. J. Neurosci 6, 355-363.

54. Guo, X., \& Wang, X.-F. (2009). Signaling cross-talk between TGF- $\beta$ /BMP and other pathways. Cell Research, 19(1), 71-88. http://doi.org/10.1038/cr.2008.302

55. Sivanandam, T. M. and Thakur, M. K. (2012). Traumatic brain injury: a risk factor for Alzheimer's disease. Neurosci. Biobehav. Rev. 36, 1376-1381.

56. Frautschy, S. A., Yang, F., Calderón, L. and Cole, G. M. (1996). Rodent models of Alzheimer's 
disease: rat A $\beta$ infusion approaches to amyloid deposits. Neurobiol. Aging 17, 311-321.

57. Byun, C. J., Seo, J., Jo, S. A., Park, Y. J., Klug, M., Rehli, M., Park, M. H. and Jo, I. (2012). DNA methylation of the $5^{\prime}$-untranslated region at+ 298 and +351 represses BACE1 expression in mouse BV-2 microglial cells. Biochem. Biophys. Res. Commun. 417, 387-392.

58. Lin, H. C., Hsieh, H. M., Chen, Y. H. and Hu, M. L. (2009). S-Adenosylhomocysteine increases $\beta$ amyloid formation in BV-2 microglial cells by increased expressions of $\beta$-amyloid precursor protein and presenilin 1 and by hypomethylation of these gene promoters. Neurotoxicology $30,622-627$

59. Gjoneska, E., Pfenning, A. R., Mathys, H., Quon, G., Kundaje, A., Tsai, L. H. and Kellis, M., 2015. Conserved epigenomic signals in mice and humans reveal immune basis of Alzheimer/'s disease. Nature 518, 365-369.

60. Werner, C. and Engelhard, K. (2007). Pathophysiology of traumatic brain injury. British Journal of Anaesthesia 99, 4-9.

61. Lee, J., Park, J., Yan, B., Kim, I., Cho, G., Jeoung, D., Kwon, Y., Kim, Y., Lee, Y., Shin, H. and Won, M. (2012). Effects of Transient Cerebral Ischemia on the Expression of DNA Methyltransferase 1 in the Gerbil Hippocampal CA1 Region. Neurochem Res, 38, 74-81.

62. Rivera, C., Voipio, J., Payne, J. A., Ruusuvuori, E., Lahtinen, H., Lamsa, K., Pirvola, U., Saarma, M. and Kaila, K. (1999). The K+/Cl- co-transporter KCC2 renders GABA hyperpolarizing during neuronal maturation. Nature 397, 251-255.

63. Lee, H. A., Hong, S. H., Kim, J. W. and Jang, I. S. (2010). Possible involvement of DNA methylation in NKCC1 gene expression during postnatal development and in response to ischemia. Journal of neurochemistry 114, 520-529.

64. Plotkin, M. D., Kaplan, M. R., Peterson, L. N., Gullans, S. R., Hebert, S. C. and Delpire, E. (1997). Expression of the $\mathrm{Na}(+)-\mathrm{K}(+)-2 \mathrm{Cl}-$ cotransporter BSC2 in the nervous system. Am J Physiol. 272, C173-C183.

65. Rivera, C., Voipio, J., Thomas-Crusells, J., Li, H., Emri, Z., Sipilä, S., Payne, J. A., Minichiello, L., Saarma, M. and Kaila, K. (2004). Mechanism of activity-dependent downregulation of the neuron-specific K-Cl cotransporter KCC2. J. Neurosci. 24, 4683-4691.

66. Shimizu-Okabe, C., Okabe, A., Kilb, W., Sato, K., Luhmann, H. and Fukuda, A. (2007). Changes in the expression of cation-Cl- cotransporters, NKCC1 and KCC2, during cortical malformation induced by neonatal freeze-lesion. Neuroscience Research,59, 288-295. 
67. Wang, F., Wang, X., Shapiro, L. A., Cotrina, M. L., Liu, W., Wang, E. W., Gu, S., Wang, W., He, X., Nedergaard, M. and Huang, J. H. (2016). NKCC1 up-regulation contributes to early posttraumatic seizures and increased post-traumatic seizure susceptibility. Brain Structure and Function 1, 1-14.

68. Viola-Saltzman, M. and Watson, N. F. (2012). Traumatic brain injury and sleep disorders. Neurol. Clin. 30, 1299-1312.

69. Shekleton, J. A., Parcell, D .L., Redman, J. R., Phipps-Nelson, J., Ponsford, J. L. and Rajaratnam, S. M. W. (2010). Sleep disturbance and melatonin levels following traumatic brain injury. Neurol. 74, 1732-1738.

70. Soria, V., Martínez-Amorós, È., Escaramís, G., Valero, J., Crespo, J.M., Gutiérrez-Zotes, A., Bayés, M., Martorell, L., Vilella, E., Estivill, X. and Menchón, J. M. (2010). Resequencing and association analysis of arylalkylamine $\mathrm{N}$-acetyltransferase (AANAT) gene and its contribution to major depression susceptibility. J. Pineal. Res. 49, 35-44.

71. Zheng, W. and Cole, P. A. (2002). Serotonin N-acetyltransferase: mechanism and inhibition. Curr. Med. Chem. 9, 1187-1199.

72. Crosio, C., Cermakian, N., Allis, C. D. and Sassone-Corsi, P. (2000). Light induces chromatin modification in cells of the mammalian circadian clock. Nat. Neurosci. 3, 1241-1247.

73. Nakahata, Y., Kaluzova, M., Grimaldi, B., Sahar, S., Hirayama, J., Chen, D., Guarente, L. P. and Sassone-Corsi, P. (2008). The NAD+-dependent deacetylase SIRT1 modulates CLOCKmediated chromatin remodeling and circadian control. Cell 134, 329-340.

74. Leeds, P. R., Yu, F., Wang, Z., Chiu, C. T., Zhang, Y., Leng, Y., Linares, G. R. and Chuang, D. M. (2014). A new avenue for lithium: intervention in traumatic brain injury. ACS Chem. Neurosci. 5, 422-433.

75. D'addario, C., Dell'Osso, B., Palazzo, M.C., Benatti, B., Lietti, L., Cattaneo, E., Galimberti, D., Fenoglio, C., Cortini, F., Scarpini, E. and Arosio, B. (2012). Selective DNA methylation of BDNF promoter in bipolar disorder: differences among patients with BDI and BDII. Neuropsychopharmacology 37, 1647-1655.

76. Dock, H., Theodorsson, A. and Theodorsson, E. (2015). DNA methylation inhibitor zebularine confers stroke protection in ischemic rats. Transl. Stroke Res. 6, 296-300.

77. Endres, M., Meisel, A., Biniszkiewicz, D., Namura, S., Prass, K., Ruscher, K., Lipski, A., Jaenisch, R., Moskowitz, M. A. and Dirnagl, U. (2000). DNA methyltransferase contributes to delayed 
ischemic brain injury. J. Neurosci. 20, 3175-3181.

78. Naim, M.Y., Friess, S., Smith, C., Ralston, J., Ryall, K., Helfaer, M. A. and Margulies, S. S. (2011). Folic acid enhances early functional recovery in a piglet model of pediatric head injury. Dev. Neurosci. 32, 466-479.

79. Sillesen, M., Bambakidis, T., Dekker, S. E., Fabricius, R., Svenningsen, P., Bruhn, P. J., Svendsen, L. B., Hillingsø, J. and Alam, H. B. (2016). Histone deactylase gene expression profiles are associated with outcomes in blunt trauma patients. J. Trauma Acute Care Surg. 80, 26-33.

80. Dębski, K. J., Pitkanen, A., Puhakka, N., Bot, A.M., Khurana, I., Harikrishnan, K.N., Ziemann, M., Kaspi, A., El-Osta, A., Lukasiuk, K. and Kobow, K. (2016). Etiology matters-Genomic DNA Methylation Patterns in Three Rat Models of Acquired Epilepsy. Scientific reports, 6.

81. Greally, J. M., \& Drake, A. J. (2017). The Current State of Epigenetic Research in Humans: Promise and Reality. JAMA pediatrics, 171(2), 103-104.

82. Mychasiuk, R., Hehar, H., Ma, I. and Esser, M. J. (2015). Dietary intake alters behavioral recovery and gene expression profiles in the brain of juvenile rats that have experienced a concussion. Front. Behav. Neurosci. 9, 17

83. Huypens, P., Sass, S., Wu, M., Dyckhoff, D., Tschöp, M., Theis, F., ... \& Beckers, J. (2016). Epigenetic germline inheritance of diet-induced obesity and insulin resistance. Nature genetics.

84. Meissner, L., Gallozzi, M., Balbi, M., Schwarzmaier, S., Tiedt, S., Terpolilli, N. A. and Plesnila, N. (2016). Temporal profile of MicroRNA expression in contused cortex after traumatic brain injury in mice. Journal of neurotrauma 33, 713-720.

85. Price, E. M., Peñaherrera, M. S., Portales-Casamar, E., Pavlidis, P., Van Allen, M. I., McFadden, D. E., \& Robinson, W. P. (2016). Profiling placental and fetal DNA methylation in human neural tube defects. Epigenetics \& chromatin, 9(1), 6.

86. Wey, H. Y., Gilbert, T. M., Zürcher, N. R., She, A., Bhanot, A., Taillon, B. D., Schroeder, F. A., Haggarty, S. J. and Hooker, J. M. (2016). Insights into neuroepigenetics through human histone deacetylase PET imaging. Science Translational Medicine, 8, pp.351ra106-351ra106.

87. Lehmann-Werman, R., Neiman, D., Zemmour, H., Moss, J., Magenheim, J., Vaknin-Dembinsky, A., ... \& Spalding, K. (2016). Identification of tissue-specific cell death using methylation patterns of circulating DNA. Proceedings of the National Academy of Sciences, 113(13), E1826-E1834. 
88. Hewitt, A., Januar, V., Sexton-Oates, A., Joo, J. E., Franchina, M., Wang, J. J., ... \& Saffery, R. (2016). DNA methylation landscape of ocular tissue relative to matched to peripheral blood. bioRxiv, 075077.

89. Wang, Z., Jiang, W., Wang, Y., Guo, Y., Cong, Z., Du, F., \& Song, B. (2015). MGMT promoter methylation in serum and cerebrospinal fluid as a tumor- ${ }^{-}$specific biomarker of glioma. Biomedical reports, 3(4), 543-548.

90. Simon, D. W., McGeachy, M. J., Bayır, H., Clark, R. S., Loane, D. J., \& Kochanek, P. M. (2017). The far-reaching scope of neuroinflammation after traumatic brain injury. Nature Reviews Neurology, 13(3), 171-191.

91. Shu, Y., Hu, Q., Long, H., Chang, C., Lu, Q. and Xiao, R., (2016.) Epigenetic Variability of CD4+ CD25+ Tregs Contributes to the Pathogenesis of Autoimmune Diseases. Clinical Reviews in Allergy \& Immunology, pp.1-13.

92. Tough, D. F., Tak, P. P., Tarakhovsky, A., \& Prinjha, R. K. (2016). Epigenetic drug discovery: breaking through the immune barrier. Nature Reviews Drug Discovery, 15(12), 835-853. 